\title{
Kinetics Model of Isothermal Pearlite Formation in a 0.4C-1.6Mn Steel
}

\author{
C. Capdevila, F. G. Caballero and C. García de Andrés
}

Department of Physical Metallurgy, Centro Nacional de Investigaciones Metalúrgicas (CENIM) Consejo Superior de Investigaciones Científicas (CSIC), Avda. Gregorio del Amo, 8. 28040 Madrid, Spain

\begin{abstract}
The present article is concerned with a theoretical and experimental study of the growth kinetics of pearlite in a $0.4 \mathrm{C}-1.6 \mathrm{Mn}$ medium carbon steels. Factors controlling the isothermal formation of this microconstituent are explored in this work. In this sense, the transition temperature between local equilibrium (LE) and no partition local equilibrium (NPLE) growth mechanisms is theoretically determined. Moreover, the nucleation of pearlite has been considered as a cementite precipitation process on a moving austenite-ferrite interface. Finally, a theoretical model is presented in this work to calculate the evolution of austenite-to-pearlite transformation with time at a very wide temperature range.
\end{abstract}

Keywords phase transformations, kinetics, steels, forging, structural

\section{1- Introduction}

Pearlite is probably the most familiar microstructural feature in the whole science of metallography. It was discovered by Sorby over 100 years ago, who assumed it to be a lamellar mixture of ferrite and cementite. Pearlite is a very common constituent of a wide variety of steels, where it provides a substantial contribution to strength, so it is not surprising that this phase has received intense study [1]. 
It is now generally agreed that during pearlite growth the alloying element distributes between the ferrite and cementite at low supersaturations (LE mechanism), and the growth is controlled by alloying element boundary diffusion. At higher supersaturations pearlite growth occurs without any partitioning of the alloying element (NPLE mechanism ) and it is controlled by carbon volume diffusion [2]. The partitioning of alloying elements has been experimentally observed by Razik et al. [3], Al-Salman et al. [4], and Chance and Ridley [5] in a number of Fe-C-Mn, Fe-C-Si, and FeC-Cr alloys. In all these studies, the partitioning was observed at low supersaturations, whereas below a characteristic temperature of the steel no partition was found.

Recent works have demonstrated that medium carbon forging steels with acicular ferrite microstructure can be manufactured at industrial scale [6-8]. The main interest of this microstructure lies in the good combination of mechanical properties that presents as compared with bainite and especially with ferritic-pearlitic microstructures. In those steels, acicular ferrite is always formed after the growth of allotriomorphic ferrite and pearlite. As a consequence, acicular ferrite transformation is inevitably influenced by previous allotriomorphic ferrite and pearlite formation. The role of the allotriomorphic ferrite to promote the formation of acicular ferrite to the detriment of bainite in a two stages heat treatment has been reported in previous works [9-10]. Thus, the amount of acicular ferrite increases as allotriomorphic ferrite is formed along the austenite grain boundaries because saturation of nucleation sites occurs. However, if pearlite transformation follows the allotriomorphic ferrite one, there will not be untransformed austenite to obtain a massive acicular ferrite transformation as temperature decrease. Therefore, a deep understanding of the decomposition of austenite in allotriomorphic ferrite and pearlite is needed in order to control the total amount of acicular ferrite present in the microstructure in medium carbon forging steels.

Recently, the austenite-to-allotriomorphic ferrite transformation has been reported by the authors [11-13]. In this work, a mathematical transformation model is presented for the purpose of simulating the austenite-to-pearlite transformation which occur during the isothermal decomposition of austenite. The model is quite general but was targeted at modern forging steels 
compositions, which tipically contain 0.4 wt.\% C, $<0.6$ wt.\%Si and 1.6 wt.\% Mn.

\section{2- Experimental Procedure}

Cylindrical dilatometric test pieces of $2 \mathrm{~mm}$ in diameter and $12 \mathrm{~mm}$ in length were machined parallel to the rolling direction of the bar. Experimental validation of the pearlite transformation kinetics model developed in this work was carried out using the heating and cooling devices of an Adamel Lhomargy DT1000 high-resolution dilatometer described elsewhere [14]. The heating device consists of a very low thermal inertia radiation furnace. The power radiated by two tungsten filament lamps is focussed on the specimen by means of a bi-elliptical reflector. The temperature is measured with a $0.1 \mathrm{~mm}$ diameter Chromel - Alumel (Type K) thermocouple welded to the specimen. Cooling is carried out by blowing a jet of helium gas directly onto the specimen surface. These devices ensure an excellent efficiency in controlling the temperature and holding time of isothermal treatments and as well as fast cooling in quenching processes.

Austenitisation conditions were fixed to avoid the influence of the austenite grain size on the kinetics of pearlite formation. Since the transformation rate of pearlite is higher the finer the prior austenite grain size (PAGS), a coarse PAGS of $76 \mu \mathrm{m}$ was selected to make easier the experimental study of the transformation kinetic of pearlite. Thus, specimens were austenitised at $1523 \mathrm{~K}$ for 1 min and subsequently isothermally transformed at temperatures ranging from 943 to $873 \mathrm{~K}$ during different times. In order to freeze the microstructure at those temperatures, specimens were quenched to room temperature by helium gas flow at a cooling rate of $200 \mathrm{~K} / \mathrm{s}$.

Specimens were polished in the usual way for metallographic examination. Nital - 2pct etching solution was used to reveal the ferrite+pearlite microstructure by optical microscopy. The volume fraction of pearlite $\left(V_{p}\right)$ was statistically estimated by a systematic manual point counting procedure

[15]. The maximum volume fraction of pearlite $\left(V_{P}^{E Q}\right)$ formed during the isothermal decomposition of austenite at 943, 933, 913 and $873 \mathrm{~K}$ was again determined by a combination of dilatometric and metallographic analysis. 
Special metallographic preparation was required to reveal the interlamellar spacing of pearlite. Deep primary etching with a solution of picric acid in isopropyl alcohol with several drops of Vilella's reagent was used to ensure that any deformed layer introduced by polishing was removed. This etching was eliminated using the 1 and $0.25 \mu \mathrm{m}$ diamond pads with almost no pressure being exerted on the sample for no longer than 3 to $4 \mathrm{~min}$. The sample was then etched again, this time lightly, and polished carefully on the 1 and $0.25 \mu \mathrm{m}$ diamond pads. Finally, a light etch was given to the sample. This preparation procedure was carefully detailed in Ref. [16]. The values of the mean true interlamellar spacing $\left(S_{o}\right)$ were derived from electron micrographs according to Underwood's intersection procedure described in Refs. [16] and [17].

The austenite-to-allotriomorphic ferrite $\left(A e_{3}\right)$ and austenite-to-pearlite $\left(A e_{1}\right)$ critical temperatures were experimentally determined by dilatometric and metallographic analysis. Initially, both temperatures were estimated from a dilatometric curve obtained by continuous cooling at a rate of $0.05 \mathrm{~K} / \mathrm{s}$. This is the rate normally used for considering quasi-equilibrium conditions [18]. Figure 1 shows the dilatometric curve obtained during austenite transformation by continuous cooling processes. Since the difficulty for monitoring separately the pearlite and proeutectoid ferrite transformation, the $\mathrm{Ae}_{1}$ temperature was more accurately determined after several isothermal heat treatments at temperatures ranging from 953 to $933 \mathrm{~K}$. Thus, a temperature of $948 \mathrm{~K}$ could be defined as the $\mathrm{Ae}_{1}$ temperature for the studied steel.

\section{3- Calculation of the interface compositions}

The interface compositions at the $\alpha \gamma$ and $\alpha \theta$ boundaries under LE and NPLE growth mechanisms have been calculated according to the method firstly reported by Kirkaldy and co-workers [19-20]. Thermodynamic data for the calculation of the compositions in ferrite $(\alpha)$, austenite $(\gamma)$ and cementite $(\theta)$ phases in a Fe-C-Mn system are listed in the Appendix. The equilibrium condition can be expressed by the equality of the chemical potentials of each element in both phases at the interface. The chemical potentials of carbon, $\mathrm{Mn}$ and Fe in ferrite, austenite and cementite are 
calculated assuming Hillert - Staffanson regular solution model, and are expressed as follows. The numbers 0, 1 and 2 denote, respectively, Fe, C and Mn. Likewise, the average mole fractions are designated as $\bar{x}_{i}$ ( $i=1$ to 2$)$, and the mol fractions in each phase as $x_{i}(i=0$ to 2$)$.

In austenite and ferrite;

$$
\begin{aligned}
& \mu_{0}=G_{0}+R T \ln x_{0}-\frac{R T}{2}\left(\varepsilon_{11} x_{1}^{2}+\varepsilon_{22} x_{2}^{2}\right)-R T \varepsilon_{12} x_{1} x_{2} \\
& \mu_{1}=G_{1}+R T \ln x_{1}+R T\left(\varepsilon_{11} x_{1}+\varepsilon_{12} x_{2}\right) \\
& \mu_{2}=G_{2}+R T \ln x_{2}+R T\left(\varepsilon_{12} x_{2}+\varepsilon_{22} x_{2}\right)
\end{aligned}
$$

and in cementite;

$$
\begin{aligned}
& \mu_{\mathrm{Fe}_{3} \mathrm{C}}=G_{\mathrm{Fe}_{3} \mathrm{C}}+\frac{3}{4} R T \ln y_{0}+\frac{3}{4}\left(1-y_{0}\right) \omega_{02} y_{2} \\
& \mu_{M_{3} \mathrm{C}}=G_{M_{3} \mathrm{C}}+\frac{3}{4} R T \ln y_{2}+\frac{3}{4}\left(1-y_{2}\right) \omega_{02} y_{0}
\end{aligned}
$$

where $\omega_{02}$ is the Fe-Mn interaction coefficient, $\varepsilon$ 's are the Wagner's interaction coefficients, and $y_{i}=4 x_{i}^{\gamma \theta} / 3$. In the case of LE mechanism the equilibrium conditions can be expressed as follows.

$\mu_{i}^{\alpha}=\mu_{i}^{\gamma}$ (i=0 to 2$)$

$4 \mu_{\mathrm{Fe}_{3} \mathrm{C}}=3 \mu_{0}^{\gamma}+\mu_{1}^{\gamma}$

$$
4 \mu_{M_{3} C}=3 \mu_{2}^{\gamma}+\mu_{1}^{\gamma}
$$

Equations (6) for carbon ( $i=1)$ and manganese $(i=2)$, and considering that $\varepsilon_{12}=\varepsilon_{12}^{\alpha}=\varepsilon_{12}^{\gamma}$, are rewritten in terms of the partition coefficients $A_{1}$ and $A_{2}$ as

$$
\begin{aligned}
& x_{1}^{\alpha \gamma}=A_{1} x_{1}^{\gamma \alpha}=\frac{\exp \left(\Delta G_{1}^{\alpha \gamma} / R T+\varepsilon_{11}^{\gamma} x_{1}^{\gamma \alpha}\right)}{1+\varepsilon_{11}^{\alpha} x_{1}^{\gamma \alpha} \exp \left(\Delta G_{1}^{\alpha \gamma} / R T\right)} x_{1}^{\gamma \alpha} \\
& x_{2}^{\alpha \gamma}=A_{2} x_{2}^{\gamma \alpha}=\frac{\exp \left(\Delta G_{2}^{\alpha \gamma} / R T+\varepsilon_{12} x_{1}^{\gamma \alpha}\right)}{1+\varepsilon_{12} x_{1}^{\gamma \alpha} \exp \left(\Delta G_{1}^{\alpha \gamma} / R T\right)} x_{2}^{\gamma \alpha}
\end{aligned}
$$


where $\Delta G_{1}^{\alpha \gamma}$ and $\Delta G_{2}^{\alpha \gamma}$ are the free energy change between austenite and ferrite for carbon and manganese, respectively. According to Hashiguchi et al. [20] the tie-line for the manganese concentration in austenite in the $\alpha / \gamma$ boundary may be expressed by,

$$
x_{2}^{\gamma \alpha}=\frac{\bar{x}_{2}\left(1-A_{1}\right) x_{1}^{\gamma \alpha}}{\left(1-A_{2}\right) \bar{x}_{1}+\left(A_{2}-A_{1}\right) x_{1}^{\gamma \alpha}}
$$

On the other hand, equation (6) for iron ( $i=0)$ can be rewritten as follows:

$$
\begin{aligned}
& \Delta G_{0}^{\alpha \gamma}+R T \ln \left[\frac{1-x_{1}^{\gamma \alpha}-x_{2}^{\gamma \alpha}}{1-x_{1}^{\alpha \gamma}-x_{2}^{\alpha \gamma}}\right]-\frac{R T}{2}\left[\left(\varepsilon_{11}^{\gamma} x_{1}^{\gamma \alpha \alpha^{2}}-\varepsilon_{11}^{\alpha} x_{1}^{\alpha \gamma^{2}}\right)+\left(\varepsilon_{22}^{\gamma} x_{2}^{\gamma \alpha^{2}}-\varepsilon_{22}^{\alpha} x_{2}^{\alpha \gamma^{2}}\right)\right] \\
& -R T \varepsilon_{12}\left(x_{1}^{\gamma \alpha} x_{2}^{\gamma \alpha}-x_{1}^{\alpha \gamma} x_{2}^{\alpha \gamma}\right)=0
\end{aligned}
$$

where $\Delta G_{0}^{\alpha \gamma}$ is the free energy change between austenite and ferrite in iron. This equation (12) contains only one unknown variable, $x_{1}^{\gamma \alpha}$, because the $x_{1}^{\alpha \gamma}$ and $x_{2}^{\alpha \gamma}$, as well as $x_{2}^{\gamma \alpha}$, are functions of $x_{1}^{\gamma \alpha}$ as expressed in equations (9), (10) and (11), respectively. Therefore, it is possible to numerically calculate the value of $x_{1}^{\gamma \alpha}$ for each temperature.

An expression for the equilibrium between cementite and austenite may be obtained from the subtraction of equation (7) from (8). Expanding the equations for the chemical potentials according to the expressions from (1) to (3), and restricting attention to low alloy steels where $\mathrm{x}_{2}<<1, \mathrm{y}_{2}<<1$, $\mathrm{x}_{0} \cong 1$ and $\mathrm{y}_{0} \cong 1$, equation (7) is approximated by

$$
\left.y_{2}=B_{2} x_{2}^{\gamma \theta}=x_{2}^{\gamma \theta} \exp \mid\left\{\Delta G_{0}^{\gamma \theta}-\Delta G_{2}^{\gamma \theta}-\omega_{02}\right\} / R T+\varepsilon_{12} x_{1}^{\gamma \theta}\right\rfloor
$$

According to Sharma et al. [21], the tie line for the Mn concentration is expressed by the following equation

$$
x_{2}^{\gamma \theta}=\frac{\bar{x}_{2}\left(4 x_{1}^{\gamma \theta}-1\right)}{3 B_{2}\left(x_{1}^{\gamma \theta}-\bar{x}_{1}\right)+4 \bar{x}_{1}-1}
$$

Expanding equation (7) according to equations (1) to (3) the following equation is yield

$$
\begin{aligned}
& \Delta G_{0}^{\theta \gamma}=R T \ln \left(\frac{1-x_{1}^{\gamma \theta}-x_{2}^{\gamma \theta}}{1-y_{2}}\right)+\frac{R T}{3} \ln x_{1}^{\gamma \theta}-\frac{R T}{2}\left(\varepsilon_{11} x_{1}^{\gamma \theta^{2}}+\varepsilon_{12} x_{2}^{\gamma \theta^{2}}\right) \\
& -\frac{R T}{3}\left(3 \varepsilon_{12} x_{1}^{\gamma \theta} x_{2}^{\gamma \theta}-\varepsilon_{11} x_{1}^{\gamma \theta}-\varepsilon_{12} x_{2}^{\gamma \theta}\right)-y_{2}^{2} \omega_{02}
\end{aligned}
$$


where $\Delta G_{0}^{\theta \gamma}$ is the free energy change between austenite and cementite phases under equilibrium conditions for iron. Since $x_{2}^{\gamma \theta}$ and $y_{2}$ are expressed as function of $x_{1}^{\gamma \theta}$, this equation is only function of $x_{1}^{\gamma \theta}$. This parameter can be solved in the same way that $x_{1}^{\gamma \alpha}$ in equation (12).

The transition from the LE to the NPLE conditions occurs when $x_{2}^{\alpha \gamma}=\bar{x}_{2}$ and $x_{2}^{\theta \gamma}=\bar{x}_{2}$ for the $\alpha \gamma$ and $\gamma / \theta$ interface, respectively. Therefore, considering that the value of $x_{2}^{\gamma \alpha}$ may be obtained from equation (10), i.e. $x_{2}^{\gamma}=\bar{x}_{2} / A_{2}$, the corresponding value of $x_{1}^{\gamma \alpha}$ at the $\alpha \gamma$ interface under NPLE consideration is derived from equation (12) assuming these values for $x_{2}^{\alpha \gamma}$ and $x_{2}^{\gamma \alpha}$. In the same way, the value of $x_{1}^{\gamma \theta}$ under NPLE mechanism at the $\gamma / \theta$ interface is calculated from equation (15) but considering $x_{2}^{\theta \gamma}=\bar{x}_{2}$ and $x_{2}^{\gamma \theta}=4 \bar{x}_{2} / 3 B_{2}$ (equation (13)).

\section{4-Theoretical determination of the maximum volume fraction of pearlite $\left(V_{P}^{E Q}\right)$}

The $V_{P}^{E Q}$ formed after the complete isothermal decomposition of austenite can be determined by applying the lever rule at the phase diagram schematically presented in Fig. 2 known as Hultgren’s extrapolation. As it was pointed out by Christian [22], pearlite starts to form at temperature below $A e_{1}$, and there is a temperature ( $T^{*}$ in Fig. 2) at which pearlite is the only decomposition product of austenite. In this sense, the maximum amount of pearlite obtained at temperatures ranging from $A e_{1}$ and $T^{*}$ could be expressed as,

$$
V_{P}^{E Q}=\frac{x_{1}^{\gamma \alpha}-x_{1}^{\gamma \theta}}{x_{1}^{\gamma \alpha}-\bar{x}_{1}}
$$

where $x_{1}^{\gamma \alpha}$ and $x_{1}^{\gamma \theta}$ are the carbon concentration in austenite which are under either LE or NPLE conditions with ferrite and cementite, respectively.

Figure 3(a) shows the section of the Fe-Mn-C system for a manganese concentration of 1.6 wt.-\%. From this figure the carbon content at the $\alpha \gamma$ and $\gamma / \theta$ interface can be easily obtained, and thus $V_{P}^{E Q}$ at different temperatures can be derived using equation (16). Figure 3(b) shows experimental 
and calculated values of $V_{P}^{E Q}$ as a function of $T$ considering either LE or NPLE conditions. According to Fig. 3(b), the formation of pearlite proceeds under LE consideration at temperatures of 943 and $933 \mathrm{~K}$, whereas no partitioning seems to be the dominant mechanism at temperature of 873

K. This behaviour is consistent with the experimental observations carried out by several authors $[1,3,23]$ in Fe-C-Mn eutectoid steels, which reported partitioning temperature of $933 \mathrm{~K}$ for manganese concentration of 1.08 wt.-\% [1], and partitioning temperature of $895 \mathrm{~K}$ form manganese concentration of 1.8 wt.- $\%$ [3,23].

\section{5- Evaluation of the transition between LE and NPLE mechanisms}

The partitioning coefficient of manganese, $K^{p}$, between ferrite and cementite is calculated using the following expression

$$
K^{p}=\frac{x_{2}^{\theta \gamma}}{x_{2}^{\alpha \gamma}}
$$

At temperatures closer than the theoretical eutectoid temperatures, $K^{p}$ is expected to have a larger value since the supersaturation of austenite is small, and the mobility of $\mathrm{Mn}$ atom is high at those temperatures. Thus, the value of $K^{p}$ decreases with temperature and reaches the unity when no partitioning condition occurs at both $\gamma / \alpha$ and $\gamma / \theta$ interfaces. Figure 4(a) shows a comparison between the measured $K^{p}$ values reported by Ridley [1] in a Fe-0.7C-1.08Mn steel, and the calculated values according to the model presented in this work. This figure suggests that the change with temperature in $K^{p}$ is successfully predicted for the model in a Fe-0.7C-1.08Mn steel. Once the reliability of the model for $K^{p}$ calculation has been proved, the variation of $K^{p}$ with isothermal temperature for the Fe-0.4C-1.6Mn steel studied in this work has been calculated. The effect of previous proeutectoid ferrite transformation has been taking into account assuming carbon enrichment in the residual austenite. Therefore, the change in the bulk carbon concentration is expressed in the following equation:

$$
\overline{x^{\prime}}=\frac{\bar{x}}{1-F}
$$


where $F$ is the volume fraction of proeutectoid ferrite transformed during the isothermal decomposition of austenite. Thus, the change in bulk carbon concentration at each isothermal temperature should be taken into account in the calculations of the interface concentrations of equation (17). Figure 4(b) shows the evolution of $K^{p}$ with the isothermal temperature in the studied steel considering the corresponding carbon enrichment in austenite due to proeutectoid ferrite formation. This figure suggests that the isothermal formation of pearlite occurs with partitioning of manganese at a temperature of $893 \mathrm{~K}$, which is consistent with the value of $\sim 895 \mathrm{~K}$ reported by Tewari and Sharma in a 0.69C-1.8Mn eutectoid steel [23].

\section{6- The onset of pearlite transformation}

Since proeutectoid ferrite is usually the first phase to develop on isothermal heat treatment, pearlite nodules nucleate on the austenite-proeutectoid ferrite $\left(\alpha_{p}\right)$ interface. It has been observed that the formation of pearlite requires the establishment of cooperative growth of ferrite and cementite [24]. The previous formation of proeutectoid ferrite enriches in carbon the surrounding austenite promoting the formation of cementite nucleus at the $\gamma / \alpha_{p}$ interface and the local reduction of carbon content in the austenite that surrounds the cementite nucleus leads to the ferrite formation of pearlite aggregate. The simultaneous ferrite and cementite formation process yields to the characteristic lamellar structure of pearlite.

Aaronson et al. [25] analysed the conditions under which nucleation is feasible at moving disordered interface boundary. The restriction that the migration rate of the $\gamma / \alpha_{p}$ boundary, $G_{\alpha \gamma}^{*}$, at which nucleation may take place must not exceed that which displaces this boundary a distance equal to the austenite lattice parameter in the time required for an embryo to develop to the critical nucleus size should be satisfied. He concluded that $G_{\alpha \gamma}^{*}$ at which a crystal of cementite can nucleate is

$$
G_{\alpha \gamma}^{*}=\frac{-a_{\gamma}^{3} D_{C}^{\gamma} x_{1}^{\gamma \alpha} \Delta G_{V}^{3}}{16(1-\cos \psi) \sqrt{\pi k_{B} T \sigma_{\alpha \theta}^{5} K}}
$$


where $a_{\gamma}$ is the austenite lattice parameter; $x_{1}^{\gamma \alpha}$ is the carbon concentration in austenite; $k_{B}$ is the Boltzmann constant; $T$ is the isothermal temperature; $D_{C}^{\gamma}$ is the carbon diffusion coefficient in austenite at the isothermal temperature; $K$ is the ratio between the volume of the double spherical cap critical nucleus and that of a sphere of the same radius; $\Delta G_{V}$ is the volume free energy change; $\cos \psi$ is the ratio between the interfacial energies of disordered $\alpha \gamma\left(\sigma_{\alpha \gamma}\right)$ and $\alpha \theta\left(\sigma_{\alpha \theta}\right)$ boundaries, and is defined as $\cos \psi=\sigma_{\alpha \gamma} / 2 \sigma_{\alpha \theta}$.

In this work, a value of $\mathrm{K}=0.0001$ has been considered [25]. Likewise, $a_{\gamma}$ has been calculated as reported by Dyson and Holmes considering the dependence of alloying elements on the lattice parameter of austenite [26]. Likewise, the value of $\Delta G_{V}$ for cementite nucleation at 913 and $873 \mathrm{~K}$ has been calculated as reported by Zener [27]. Values of $a_{\gamma}$ and $\Delta G_{V}$ at 913 and $873 \mathrm{~K}$ are listed in Table 1.

Calculations of $D_{C}^{\gamma}$ have been carried out according to Bhadeshia [28]. The author considers both the kinetic and equilibrium thermodynamic behaviour of carbon in austenite. These calculations takes also into account the concentration dependence of the activity of carbon in austenite, and the repulsive interactions between the nearest neighbouring carbon atoms located in octahedral interstitial sites. Thus, $D_{C}^{\gamma}$ is calculated by two factors: one of them is a concentration dependent factor and the other one is independent

$$
D_{C}^{\gamma}=\xi(\theta) \frac{k_{B} T}{h}\left(\frac{\lambda^{2}}{3 \gamma_{m}}\right) \exp \left\{-\frac{\Delta G *}{k_{B} T}\right\}
$$

where $\xi(\theta)$ is the carbon concentration dependent factor obtained according to Bhadeshia's calculations [28] and takes values listed in Table $1 ; \Delta G^{*}$ is the activation energy for diffusion; $\gamma_{m}$ is an activity coefficient assumed constant; $\lambda$ is the distance between the $\{002\}$ austenite planes and $h$ is the Planck's constant. Bhadeshia [28] found that $\Delta G^{*} / k_{B}=21230 \mathrm{~K}$ and $\ln \left(\gamma_{m} / \lambda^{2}\right)=31.84$. The values of $D_{C}^{\gamma}$ for temperatures of $913 \mathrm{~K}$ and $873 \mathrm{~K}$ are also listed in Table 1. 
Finally, assuming that the $\gamma / \alpha_{p}$ interface is a planar disordered boundary and of infinite extent, the velocity of the moving interface ( $G_{\alpha \gamma}$ ) diminishes with time as follows,

$$
G_{\alpha \gamma}=\frac{\alpha_{1}}{2 t^{1 / 2}}
$$

where $\alpha_{1}$ is the one-dimensional parabolic growth rate constant and $t$ represents the growth time. Bradley and Aaronson [29] reported a comparison between measured allotriomorphic ferrite growth kinetics data in a Fe-C-Mn with the predictions of three different models. They concluded that paraequilibrium model is the most satisfactory of those available. The value of $\alpha_{1}$ under paraequilibrium conditions can be obtained by numerical solution from the equation [11,22]

$$
\left(\frac{\pi}{4 D_{C}^{\gamma}}\right)^{1 / 2} \alpha_{1} \exp \left(\frac{\alpha_{1}^{2}}{4 D_{C}^{\gamma}}\right) \operatorname{erfc}\left(\frac{\alpha_{1}}{2 \sqrt{D_{C}^{\gamma}}}\right)=\frac{x_{1}^{\gamma \alpha}-\bar{x}_{1}}{x_{1}^{\gamma \alpha}-x_{1}^{\alpha \gamma}}=\Omega
$$

The values of $\Omega$ and $\alpha_{1}$ for temperatures of 913 and $873 \mathrm{~K}$ are listed in Table 1.

A comparison between equations (19) and (21) allows us to evaluate the time required to start to form pearlite during the isothermal decomposition of austenite in the steel studied $\left(t^{*}\right)$. However, it is necessary to evaluate the value of $\sigma_{\alpha \theta}$ and $\sigma_{\alpha \gamma}$ in the studied steel in order to determine $G_{\alpha \gamma}^{*}$. An estimation of the value of this interface energy is yield by measuring the interlamellar spacing of pearlite as shown below.

\section{7- Calculation of ferrite-cementite interfacial energy $\left(\sigma_{\alpha \theta}\right)$}

When the growth rate of pearlite is controlled by the bulk diffusion of carbon in austenite, Zener [27] proposed the following relationship for the interlamellar spacing, $S_{o}$, and the theoretical critical spacing at zero growth rate, $S_{c}$, based in the maximum growth rate criterion:

$$
S_{o}=2 S_{C}=\frac{4 A e_{1} \sigma_{\alpha \theta}}{\Delta H_{v}\left(A e_{1}-T\right)}
$$


where $T$ is the formation temperature; $A e_{1}$ is the eutectoid temperature; $\sigma_{\alpha \theta}$ is the interfacial energy per unit area of the ferrite-cementite lamellar boundary in pearlite, and $\Delta H_{v}$ is the change in enthalpy of transformation per unit volume.

However, when the partitioning of the substitutional alloying elements is substantial during the growth of pearlite, boundary diffusion of the alloying elements may control the growth rate of pearlite. In that case, the maximum growth rate criterion of Zener gives an expression for $S_{o}$ as a function of the pearlite formation temperature as follows:

$$
S_{o}=\frac{3 A e_{1} \sigma_{\alpha \theta}}{\Delta H_{v}\left(A e_{1}-T\right)}
$$

Figure 5 shows scanning electron micrographs of pearlite obtained after full decomposition of austenite at 943, 933, 913 and $873 \mathrm{~K}$. The $S_{o}$ value at these temperatures has been measured from micrographs in Fig. 5. Figure 6 represent the variation of the interlamellar spacing as a function of the temperature formation. The $\sigma_{\alpha \theta}$ value is derived from equation (23) or (24) depending on the rate controlling mechanism that occurs at each temperature. A value of $\Delta H_{V}=6.7 \times 10^{8} \mathrm{~J} \mathrm{~m}^{-3}$ [27] has been considered in the determination of $\sigma_{a \theta}$. In this sense, a value of $\sigma_{\alpha \theta}=0.68 \pm 0.06 \mathrm{~J} \mathrm{~m}^{-2}$ has been achieved. This value is consistent with that attained by Kirchner et al. [30] $\left(\sigma_{\alpha \theta}=0.6 \mathrm{~J} \mathrm{~m}^{-2}\right)$ for Fe-C-Mn steels.

Finally, in order to determine $G_{\alpha \gamma}^{*}$, a value of $\cos \psi=0.08$ in equation (19) has been obtained from the interfacial energies reported by Reed and Bhadeshia [31] $\left(\sigma_{\alpha \gamma}=0.1 \mathrm{~J} \mathrm{~m}^{-2}\right)$ and the above derived $\sigma_{\alpha \theta}\left(\sigma_{\alpha \theta}=0.68 \mathrm{~J} \mathrm{~m}^{-2}\right)$. Figure 7 shows a comparison between calculated and experimental t* values for the studied steel. From this figure it can be concluded that a good agreement between experimental and predicted values of $t^{*}$ exists.

\section{8- Modelling of austenite-to-pearlite transformation}


Puls and Kirkaldy [32], in their review on the pearlite reaction showed the following expression for the pearlite growth velocity based on Hillert's theory when the growth rate of pearlite is controlled by the bulk diffusion of carbon in austenite ahead of the interface or NPLE condition [33]:

$$
G_{N P L E}=\frac{D_{C}^{\gamma}\left(x_{1}^{\gamma \alpha}-x_{1}^{\gamma \theta}\right)}{g\left(x_{1}^{\theta \gamma}-x_{1}^{\alpha \gamma}\right)} \frac{S_{o}}{S_{\alpha} S_{\theta}}\left[1-\frac{S_{C}}{S_{o}}\right]
$$

where $g$ is a geometric factor equal to $0.72 ; D_{C}^{\gamma}$ is the carbon diffusion coefficient in austenite; $S_{c}$ is the theoretical critical spacing at zero growth rate; $S_{\theta}$ and $S_{\alpha}$ are the thickness of cementite and ferrite lamellae, respectively. The ratio between $S_{\theta}$ and $S_{\alpha}$ was assumed to be 7 .

The pearlite growth in the partitioned reaction may be controlled by the alloying element interface diffusion (boundary diffusion model) [34]. The growth rate, in that case is expressed as follows:

$$
G_{L E}=12 K^{P} D_{B}^{\gamma} \delta \frac{\left(x_{2}^{\gamma \alpha}-x_{2}^{\gamma \theta}\right)}{\bar{x}_{2}} \frac{1}{S_{\alpha} S_{\theta}}\left[1-\frac{S_{C}}{S_{0}}\right]
$$

where $K^{P}$ is the boundary segregation coefficient calculated according to equation (17); $D_{B}^{\gamma}$ is the boundary diffusion coefficient of substitutional alloying element, i.e. Mn; and $\delta$ is the thickness of the boundary. Assuming that the activation energy for boundary diffusion of $\mathrm{Mn}$ is the half of that for self-diffusion and the boundary thickness is $0.25 \mathrm{~nm}, D_{B}^{\gamma} \delta$ can be expressed as $12.5 \times 10^{-14} \exp (-$ 139108/RT) in $\mathrm{m}^{3} \mathrm{~s}^{-1}$ [35].

The overall transformation kinetics of pearlite was described by Johnson-Mehl-Avrami theory, and here is adapted for hemispherical particles of radius $r_{p}$ nucleating at the $\gamma / \alpha_{p}$ boundaries at a rate $I$ after incubation time $\tau$. The particles grow with a constant rate $G_{L E}$ or $G_{N P L E}$ depending on the temperature range studied. In order to determine the volume fraction of pearlite formed at a given temperature, a series of planes parallel to the boundary and spaced a distance $d y$ apart are considered. If the radius of a particle exceeds the distance $y$ of a plane from the boundary, then the area of intersection of that particle with the plane is determined as Fig. 8 illustrates. The total of such areas of intersection on one plane for all particles growing from the boundary is the extended area of transformation on that plane. 
If the area of intersection is $\pi r_{p}^{2}=\pi\left(G^{2}(t-\tau)^{2}-y^{2}\right)$, the change in extended area of pearlite on one plane due to particles emanating from one boundary in the time interval from $\tau$ to $\tau+\mathrm{d} \tau$ can be expressed as:

$\left.d O_{e}=\pi O_{b} I \mid G^{2}(t-\tau)^{2}-y^{2}\right] d \tau$

for $r_{p}>y$, otherwise $d O_{e}=0$. The $O_{b}$ is the total area of the plane. Assuming that $I$ is constant, substituting $\phi=y / G t$, and integrating over all incubation times, from $\tau=0$ to $\tau=t-(y / G)$, the total extended area of pearlite on one plane is obtained:

$O_{e}=\frac{\pi}{3} O_{b} I G^{2} t^{3}\left(1-3 \phi^{2}-2 \phi^{3}\right)$

The actual area $O_{p}$ which intersecs the plane $O_{b}$ will be smaller than the extended area, since the extended area includes a fraction $\left[1-\left(O_{p} / O_{b}\right)\right]$ of 'phantom' area which has already transformed to pearlite. The relationship between the extended area and the actual area $O_{p}$ is then given by [36]

$$
\frac{O_{e}}{O_{b}}=\ln \left(1-\frac{O_{p}}{O_{b}}\right)
$$

If it is assumed that there is no interference from particles emanating from other $\gamma / \alpha_{p}$ boundaries, then the total volume of pearlite originating from one boundary, $V_{b}$, can be calculated by integrating the actual area over all the planes $y$. Thus,

$V_{b}=O_{b} G t\left[\int_{0}^{1} 1-\exp \left\{-\frac{\pi}{3} I G^{2} t^{3}\left(1-3 \phi^{2}-2 \phi^{3}\right)\right\} d \phi\right]=O_{b} G t f(G, I, t)$

If $V$ is the total volume of the assembly and $S_{V}$ the $\gamma / \alpha_{p}$ boundary surface area per unit volume, the total extended volume transformed from all boundaries $\left(V_{e}\right)$ can be calculated from the following equation not considering the hard impingement or overlapping of regions emanating from different boundaries:

$\frac{V_{e}}{V}=S_{V} G t f(G, I, t)$ 
This can be converted into the actual volume of pearlite, $V_{p}$, using the following equation (related to equation (31))

$$
\frac{V_{p}}{V_{p}^{E Q}}=1-\exp \left(-\frac{V_{e}}{V}\right)
$$

where $V_{P}^{E Q}$ is the maximum amount of pearlite obtained after the complete isothermal decomposition of austenite calculated according to equation (16). It follows that the extent of the pearlite reaction, $\xi$, is therefore:

$$
\xi=\frac{V_{p}}{V_{P}^{E Q}}=1-\exp \left\{-S_{V} G t f(G, I, t)\right\}
$$

The $\gamma / \alpha_{p}$ boundary area per unit volume, $S_{V}$, can be estimated assuming that proeutectoid ferrite nucleates at the austenite grain surface under the condition of site saturation and grow within the austenite grains. Such assumption gives [37]

$$
S_{V}=S_{\gamma}(1-F)^{2 / 3}
$$

where $S_{\gamma}$ is the austenite grain boundary per unit volume; $F$ is the volume fraction of proeutectoid ferrite transformed before pearlite reaction starts. Assuming that austenite grains have a tetrakaidecahedra geometry [36], $\mathrm{S}_{\gamma}=3.35 / \mathrm{d}_{\gamma}$ where $\mathrm{d}_{\gamma}$ is the austenite grain diameter.

The nucleation rate of pearlite on $\not / \alpha_{p}$ boundary per unit area $(I)$ has been calculated as reported by Reed and Bhadeshia [31]. The values of the parameters used in the calculation of pearlite volume fraction as a function of time at 913 and $873 \mathrm{~K}$ are listed in Table 2.

Figure 9 shows the experimental and predicted evolution of $V_{p}$ during the isothermal decomposition of austenite at 913 and $873 \mathrm{~K}$, assuming either full partition of alloying elements (boundary diffusion) or carbon partition (bulk diffusion) between austenite and pearlite. It could be concluded from this figure that partitioning of manganese is the dominant mechanism controlling pearlite growth during isothermal decomposition of austenite at $913 \mathrm{~K}$, whereas partitioning of carbon occurs at $873 \mathrm{~K}$. 


\section{9- Conclusions}

1. The kinetics of austenite-to-pearlite transformation has been described in a wide temperature range for a $0.4 \mathrm{C}-1.6 \mathrm{Mn}$ forging steel. The proposed kinetic model successfully considers the change in pearlite growth mechanisms at low and high supersaturation.

2. The transition temperature between LE and NPLE growth mechanisms has been theoretically determined at $893 \mathrm{~K}$ for a $0.4 \mathrm{C}-1.6 \mathrm{Mn}$. This result is consistent with experimental measurements of this temperature carried out by Tewari and Sharma [23] in a similar steel.

3. The onset of pearlite transformation occurs when carbon concentration of saturated austenite falls into the denominated Hultgren's extrapolation of the phase diagram, and simultaneously, the $\gamma / \alpha_{p}$ interface progresses at a rate lower than $G_{\alpha \gamma}^{*}$ to allow cementite precipitation on a moving interface. The mathematical procedure presented in this work successfully predicts the incubation time for pearlite formation.

4. The overall transformation kinetics of pearlite described by Johnson-Mehl-Avrami theory has been successfully adapted for hemispherical particles nucleating at the $\gamma / \alpha_{p}$ boundaries. The assumption of pearlite growth under LE at temperatures above $898 \mathrm{~K}$ and under NPLE at temperatures below has been confirmed by means of the good agreement between experimental and calculated results in the studied steel.

5. The model presented in this work is based on physical and metallurgical principles of phase transformations. Although the proposed model has only been validated for a $0.4 \mathrm{C}-1.6 \mathrm{Mn}$ steel, in principle this model is able to predict the isothermal decomposition of austenite in pearlite for a wide range of steels. This model is different to those empirical and semiempirical models created by fitting equations to experimental data.

\section{Acknowledgements}

The authors acknowledge financial support from Spanish Ministerio de Cioencia y Tecnología (MAT2001-1617). C. Capdevila would like to express his gratitude to the Consejo Superior de 
Investigaciones Cientifícas for financial support as a Post-Doctoral contract (I3P PC-2001-1). F.G. Caballero would like to thank the Consejería de Educación. D.G. de Investigación de la Comunidad Autónoma de Madrid (CAM) for the financial support in the form of a Postdoctoral Research Grant.

\section{Appendix}

The thermodynamic parameters for this work are taken from Uhrenius' tabulation [38] which has been adapted to a sub-regular solution model.

$$
\begin{aligned}
& \Delta G_{0}^{\gamma \alpha}=8933-14.41 T+12.08 \times 10^{-3} T^{2}-11.51 \times 10^{-6} T^{3}+5.23 \times 10^{-9} T^{4} \quad(\mathrm{~T}<1000 \mathrm{~K}) \\
& \Delta G_{0}^{\gamma \alpha}=71659-216.8 T+24.77 \times 10^{-2} T^{2}-12.66 \times 10^{-5} T^{3}+24.4 \times 10^{-9} T^{4}(\mathrm{~T} \geq 1000 \mathrm{~K}) \\
& \Delta G_{2}^{\gamma \alpha}=-20520+4.088 T+1500 S_{\text {mag }}^{\alpha} \\
& \Delta G_{1}^{\gamma \alpha}=-65562+32.949 T \\
& \Delta G_{0}^{\gamma \theta}=1.332 \times 10^{4}-64.718 T+7.481 T \ln T-G^{g r} \\
& \Delta G_{2}^{\gamma \theta}=-14263+10 T-G^{g r}
\end{aligned}
$$

The values of $S_{m a g}^{\alpha}$ and $\mathrm{G}^{\mathrm{gr}}$ are, respectively,

$$
\begin{array}{lr}
S_{\text {mag }}^{\alpha}=-11.91 \times 10^{-4} T+8.27 \times 10^{-6} T^{2}-15.1 \times 10^{-9} T^{3}+12.86 \times 10^{-12} T^{4} & (\mathrm{~T}<1075 \mathrm{~K}) \\
S_{\text {mag }}^{\alpha}=208.24-36710 / T-23.973 \ln T & (1075 \leq \mathrm{T} \leq 1500 \mathrm{~K}) \\
S_{\text {mag }}^{\alpha}=7.87-4.18 \times 10^{-4} T & (1500 \mathrm{~K}>\mathrm{T}) \\
G^{g r}=15383.3-6.402 T &
\end{array}
$$

The Wagner interaction coefficients for ferrite and austenite phases are listed as follow

$$
\begin{aligned}
& \varepsilon_{11}^{\gamma}=4.786+5066 / T \\
& \varepsilon_{11}^{\alpha}=1.3 \\
& \varepsilon_{22}^{\gamma}=2.406-175.6 / T \\
& \varepsilon_{22}^{\alpha}=3.082-4679 / T+1509.8 S_{\text {mag }}^{\alpha} / T
\end{aligned}
$$


$\varepsilon_{12}=-4811 / T$

Finally, the Fe-Mn interaction coefficient is $\omega_{02}=8351-15.19 T$

\section{References}

[1] Ridley, N., Metall. Trans., 1984, 16A, 1019.

[2] Sharma, R. C., Purdy, G.C., Kirkaldy, J. S., Metall. Trans., 1979, 10A ,1129.

[3] Razik, N. A., Lorimer, G. W., Ridley, N., Acta Metall., 1974, 22, 1249.

[4] Al-Salman, S. A., Lorimer, G. W., Ridley, N., Acta Metall., 1979, 27, 1391.

[5] Chance, J., Ridley, N., Metall. Trans., 1981, 12A, 1205.

[6] Madariaga, I., Gutierrez, I., Garcia de Andres, C., Capdevila, C., Scripta Mater., 1999, 41, 229.

[7] Madariaga, I., Gutierrez, I., Acta Mater., 1999, 47, 951.

[8] García de Andrés, C., Capdevila, C., Madariaga, I., Guitérrez, I., Scripta Mater., 45, 2001, 709.

[9] García de Andrés, C., Capdevila, C., Caballero, F.G., in Proc. Congreso Nacional de Tratamientos Térmicos y de Superficie TRATERMAT 98, M. Carsi (ed), CENIM-CSIC, Madrid, Spain, 1998, p. 135.

[10]Babu, S.S., Bhadeshia, H.K.D.H., Svensson,L.E-, J. Mater. Sci. Lett., 1991, 10,142.

[11]Capdevila,C., García de Andrés, C., Caballero, F.G., Metall. Trans., 32A, 2001, 661.

[12]Capdevila, C., García de Andrés, C., Caballero, F.G.,, Scripta Mater., 44, 2001, 593.

[13] Capdevila, C., Caballero, F.G., García de Andrés, C., Scripta Mater., 44, 2001, 129.

[14] García de Andrés, C., Caruana, G., Alvarez, L.F., Mat. Sci. Eng., 1998, A241, 211.

[15] Vander Voort, G.F., “Metallography”, McGraw-Hill, NY, 1984, p. 27.

[16]Caballero,F.G., García de Andrés, C., Capdevila, C., Mat. Character., 45, 2000, 111.

[17]Underwood, E.E., ‘Quantitative Stereology’, Addison-Wesley, Reading, 1970, p.73.

[18] García, C., Alvarez, L.F., Carsí, M., Welding International, 1982, 6, 612. 
[19]Kirkaldy, J.S., Thomson, B.A., Bagains, E.A., 'Hardenability concepts with applicacitions to steel', D.V. Doane and J.S. Kirkaldy (eds), Trans. AIME, Warrendale, Pennsylvania, 1978, p. 83.

[20]Hashiguchi,K.,Kirkaldy,J.S., Fukuzumi, T., Pavaskar, V., CALPHAD, 8, 1984, 173.

[21] Sharma, R.C., Purdy, G.R., Kirkaldy, J.S., Metall. Trans., 1979, 10A, 1129.

[22]Christian, J.W., “Theory of Transformations in metals and alloys”, Part I, $2^{\text {nd }}$ edn, Pergamon Press,Oxford, 1975, p.482.

[23] Tewari, S.K., Sharma, R.C., Metall. Trans., 16A, 1985, 597.

[24]Hillert, M., 'The decomposition of Austenite by Diffusional Processes', F. Zackay and H.I. Aaronson (eds), Interscience, NY, 1962, 197.

[25] Aaronson,H.I.,Plichta,M.R.,Franti,G.W.,Russell,K.C.,Metall. Trans., 9A, 1978, 363.

[26]Dyson D.J., Holmes, B., JISI, 1970, 208, 469.

[27]Zener, C., Trans. AIME, 1946, 167, 550.

[28] Bhadeshia, H.K.D.H., Metal Sci., 1981,15, 477.

[29]Bradley, J.R., Aaronson, H.I., Metall. Trans., 1981, 12A, 1729.

[30]Kirchener, H.O.K., Mellor, B.G., Chadwick, G.A., Acta Met., 1978, 26, 1023.

[31] Reed, R.C., Bhadeshia, H.K.D.H., Mat. Sci. Technol., 1992, 8, 421.

[32]Puls, M.P., Kirkaldy, J.S., Metall. Trans., 1972, 3, 2777.

[33] Hillert, M., Jernkont. Ann., 1957, 141, 757.

[34]Kirkaldy, J.S., Metall. Trans., 1973,4, 2327.

[35] Krishtal, M.A., “Diffusion processes in iron alloys”, Israel program for scientific translations, Jerusalem, 1970, p. 90.

[36]Cahn, J.W., Acta Metall., 1956, 4, 449.

[37]Unemoto, M., Hiramatsu, A., Moriya, A., Watanabe, T., Nanba, S., Nakajima, N., Anan, G., Higo, Y., ISIJ, 1992, 32, 306. 
[38]Uhrenius, B., 'Hardenability concepts with applicacitions to steel', D.V. Doane and J.S. Kirkaldy (eds), Trans. AIME, Warrendale, Pennsylvania, 1978, p. 28.

\section{Figure captions}

Fig. $1-A e_{3}$ and $A e_{1}$ critical temperatures marked on the cooling segment of a dilatometric curve obtained by continuous cooling at $0.05 \mathrm{~K} / \mathrm{s}$.

Fig. 2- Schematical equilibrium diagram for pearlite transformation showing the extrapolated phase boundaries (Hultgren's extrapolation).

Fig. 3- Calculated (a) isopleth of the Fe-C-Mn system (Mn content of 1.6 wt.-\%), and (b) evolution of pearlite volume fraction under LE and NPLE.

Fig. 4- Calculated $\mathrm{K}^{\mathrm{P}}$ coefficient (a) as compared with experimentally obtained in a 0.7C-1.08Mn eutectoid steel, and (b) in the studied steel.

Fig. 5- SEM images obtained after full decomposition of austenite at (a) 943 K, (b) 933 K, (c) 913 $\mathrm{K}$, and (d) $873 \mathrm{~K}$.

Fig. 6- Evolution of $S_{o}$ as a function of the undercooling below $\operatorname{Ae}_{1}(\Delta T)$.

Fig. 7- Comparison between calculated (open circles) and experimental (black filled squares) t* values. 
Fig. 8- Schematic illustration of semi-spherical particles growing from a $\alpha / \gamma$ boundary and intersecting a parallel plane a distance y form the boundary.

Fig. 9- Experimental and predicted evolution of $\mathrm{V}_{\mathrm{P}}$ during isothermal decomposition of austenite at (a) $913 \mathrm{~K}$ and (b) $873 \mathrm{~K}$.

\section{Tables}

Table 1. Calculated values of $a_{\gamma} \Delta \mathrm{G}_{\mathrm{v}}, \xi(\theta), D_{C}^{\gamma}, \Omega$ and $\alpha_{1}$ parameters .

\begin{tabular}{ccccccc}
\hline$T,(\mathrm{~K})$ & $a_{\gamma} \mu \mathrm{m}$ & $\Delta \mathrm{G}_{\mathrm{v},} \mathrm{J} \mathrm{m}^{-3}$ & $\xi(\theta)$ & $D_{C}^{\gamma}, \mathrm{m}^{2} \mathrm{~s}^{-1}$ & $\Omega$ & $\alpha_{1}, \mathrm{~m} \mathrm{~s}^{-1 / 2}$ \\
\hline 913 & 3.626 & $2.5 \times 10^{7}$ & 0.016 & $12.0 \times 10^{-14}$ & 0.60 & $0.4 \times 10^{-7}$ \\
873 & 3.622 & $5.8 \times 10^{7}$ & 0.023 & $5.8 \times 10^{-14}$ & 0.70 & $1.2 \times 10^{-6}$ \\
\hline
\end{tabular}

Table 2. Values of $I_{b}, S_{V}, G_{N P L E}$ and $G_{L E}$ at the two tested tempeatures.

\begin{tabular}{ccccc}
\hline $\mathrm{T}, \mathrm{K}$ & $\mathrm{G}_{\mathrm{NPLE}}, \mathrm{m} \mathrm{s}^{-1}$ & $\mathrm{G}_{\mathrm{LE}}, \mathrm{m} \mathrm{s}^{-1}$ & $\mathrm{I}, \mathrm{m}^{-2} \mathrm{~s}^{-1}$ & $\mathrm{~S}_{\mathrm{V}}, \mathrm{m}^{-1}$ \\
\hline 913 & $11.1 \times 10^{-6}$ & $1.2 \times 10^{-6}$ & $1.93 \times 10^{7}$ & 32564 \\
873 & $13.3 \times 10^{-6}$ & $1.5 \times 10^{-6}$ & $5.8 \times 10^{6}$ & 35602 \\
\hline
\end{tabular}


Figures

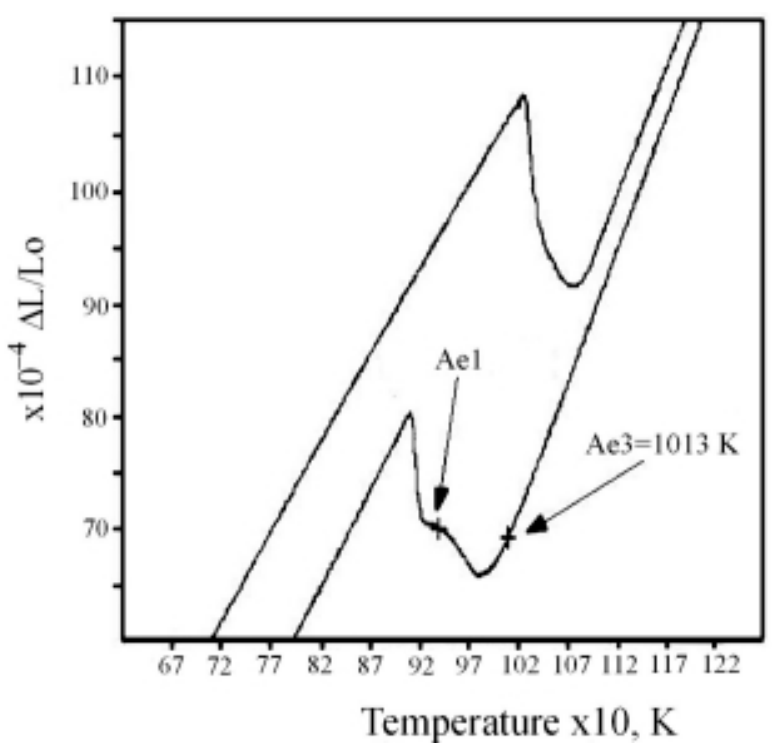

Figure 1

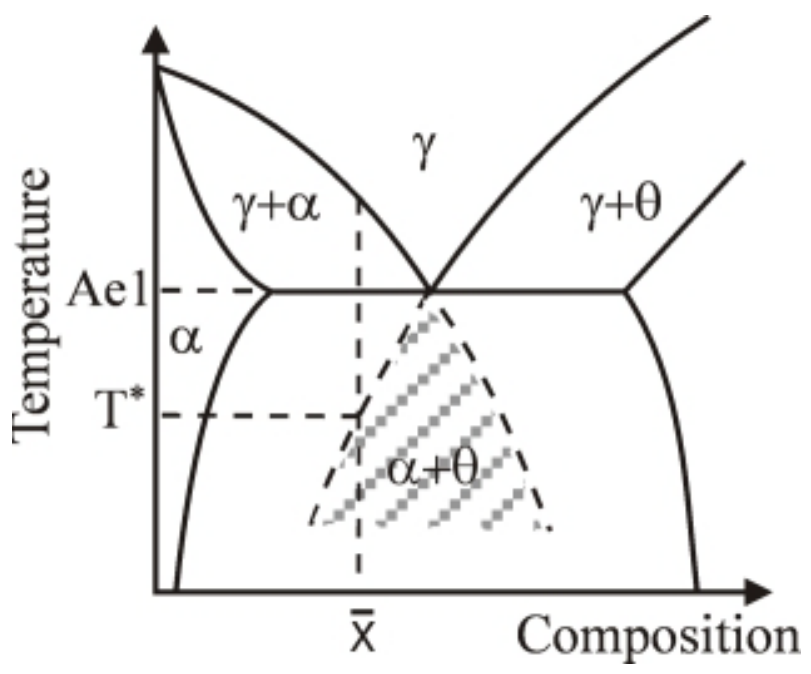

Figure 2 


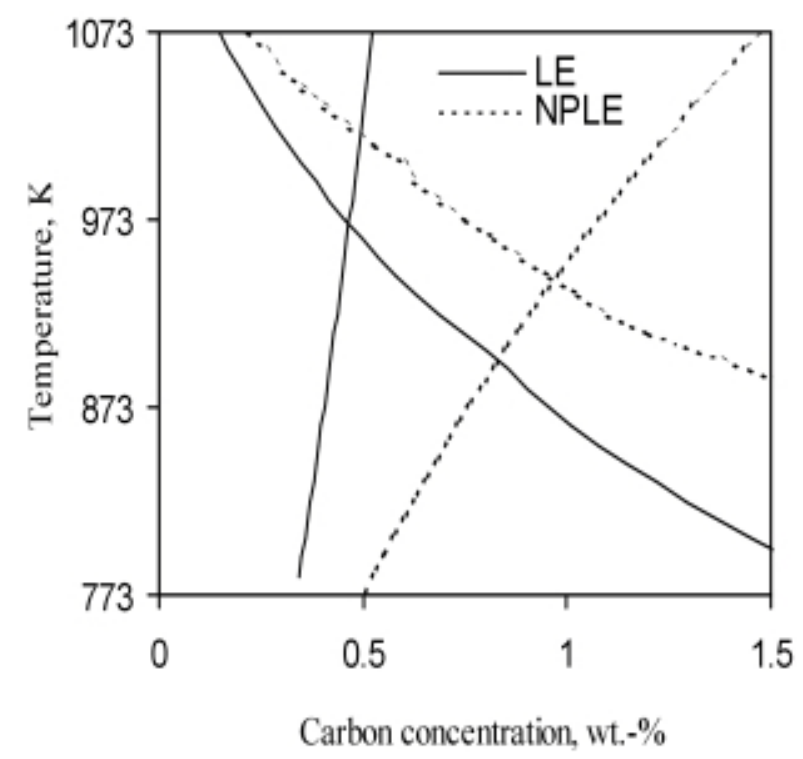

Figure 3(a)

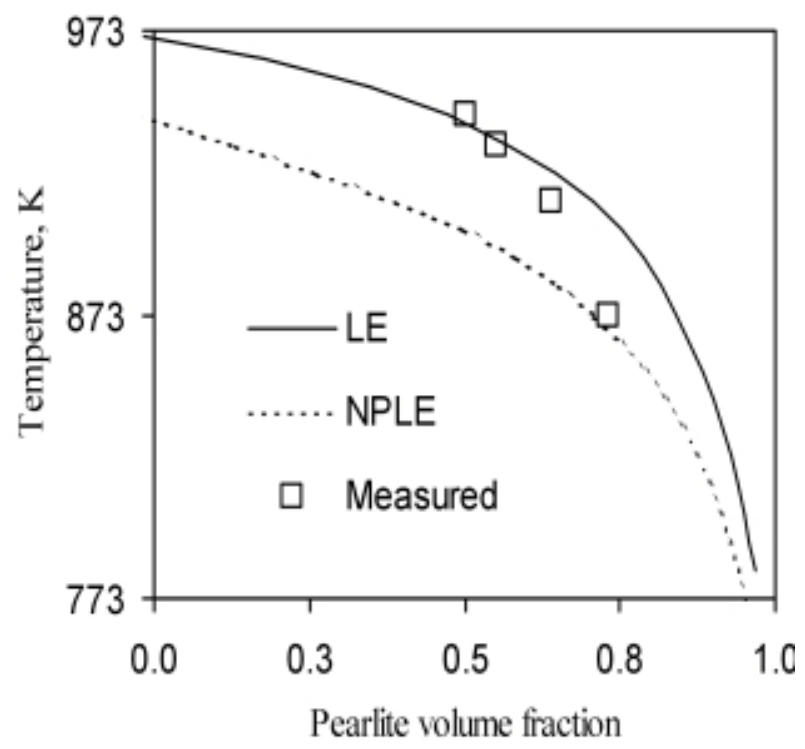

Figure 3(b) 


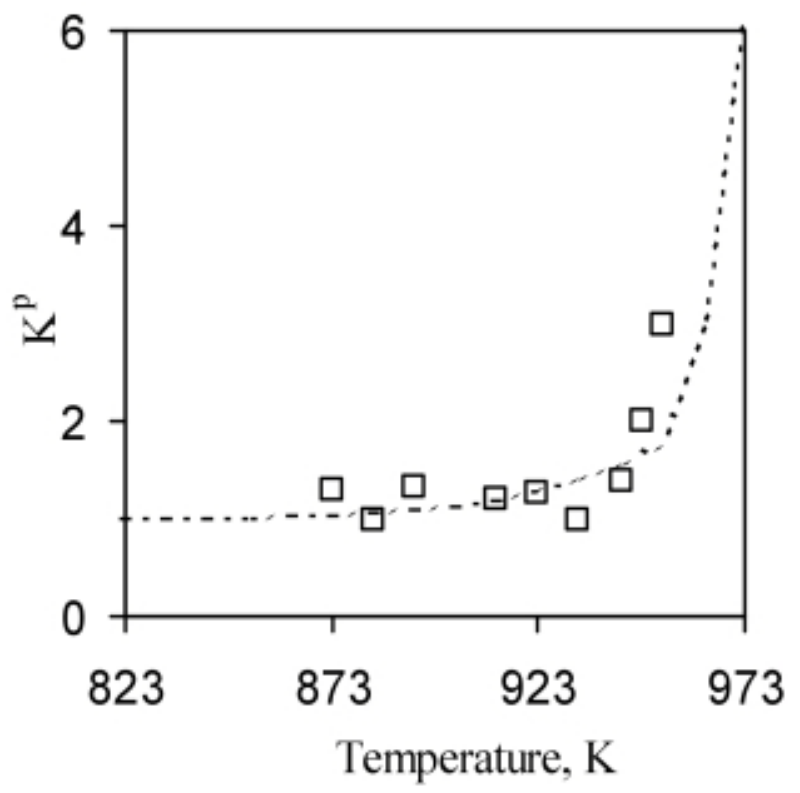

Figure 4(a)

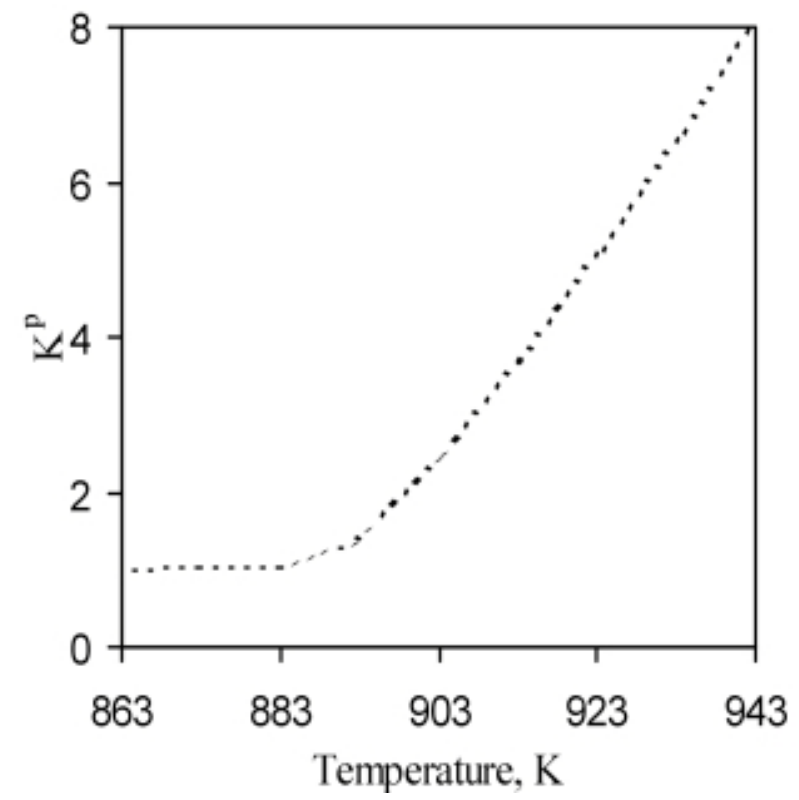

Figure 4(b) 


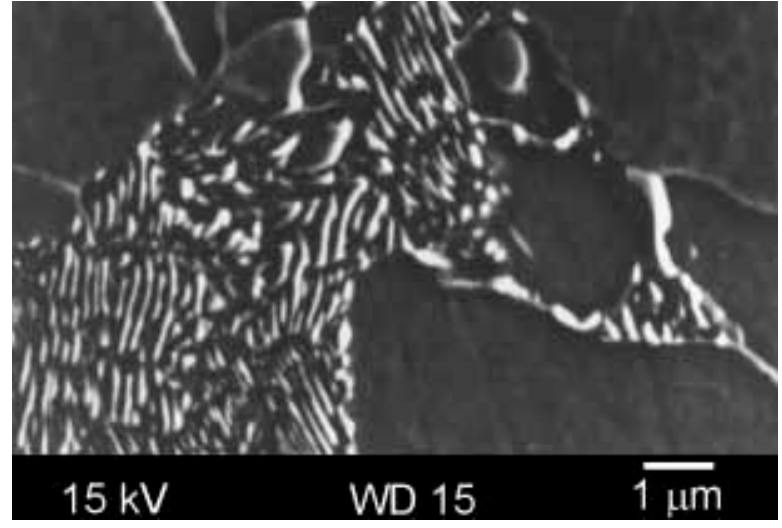

Figure 5(a)

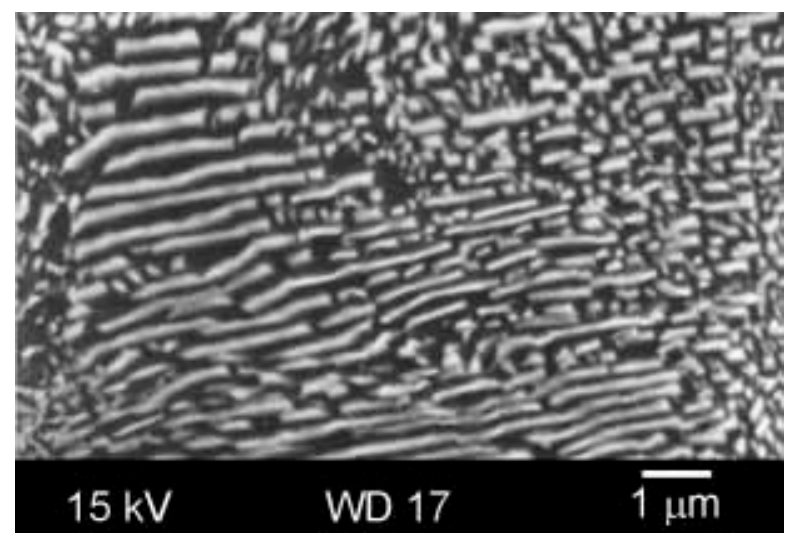

Figure 5(c)

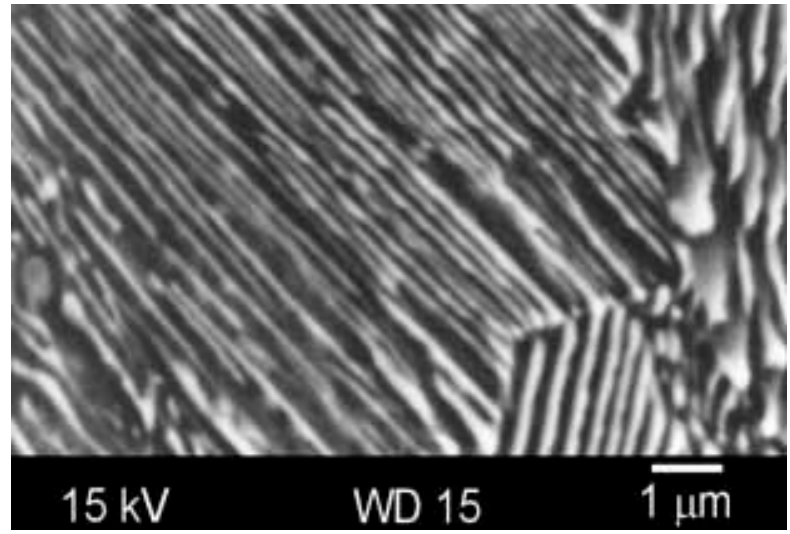

Figure 5(b)

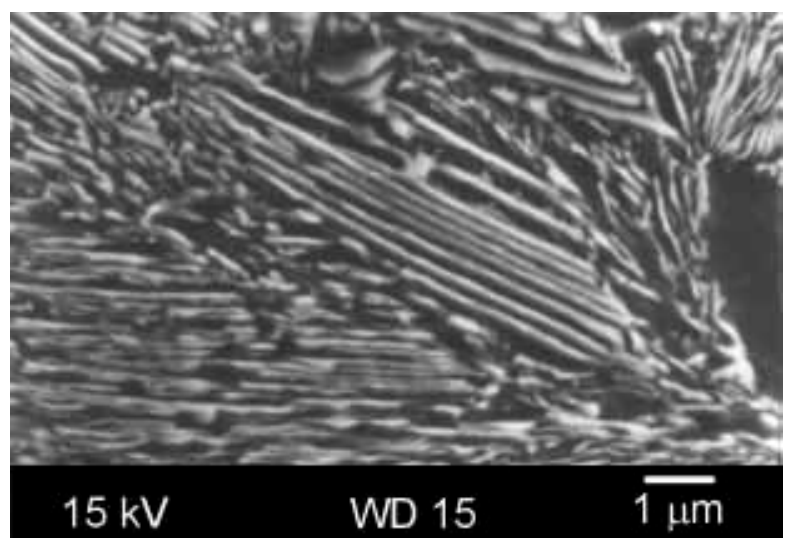

Figure 5(d) 


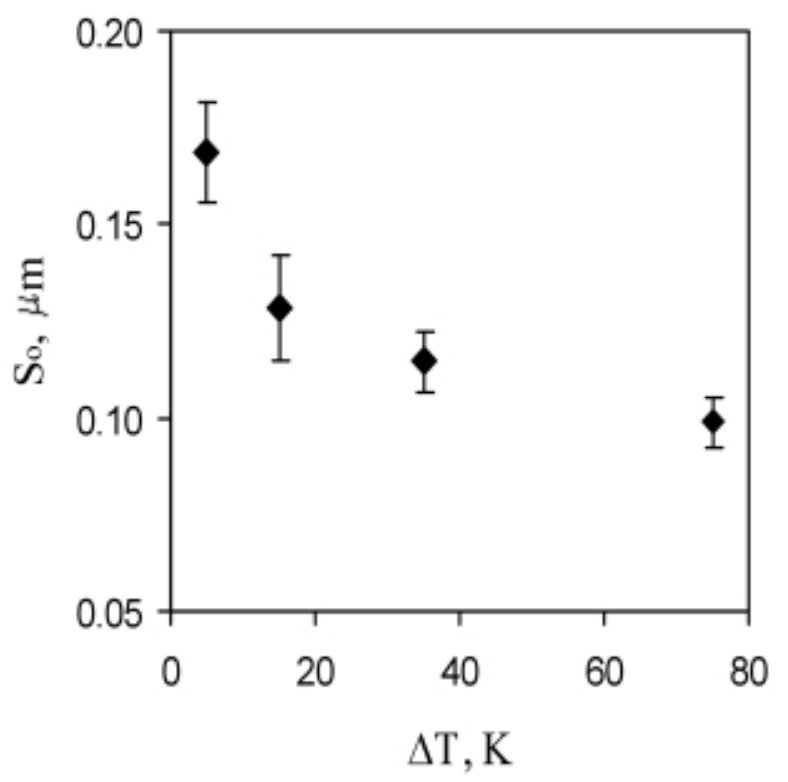

Figure 6

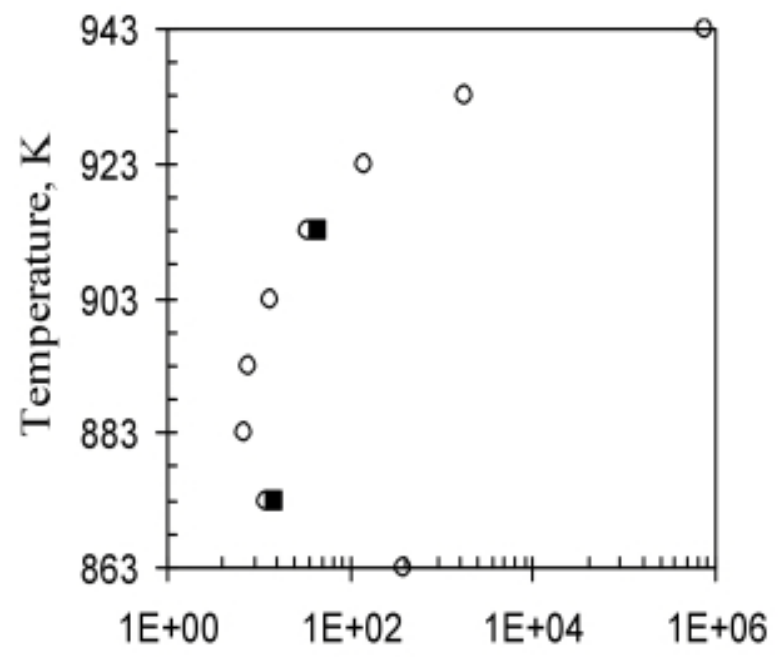

Time, $\mathrm{s}$

Figure 7 


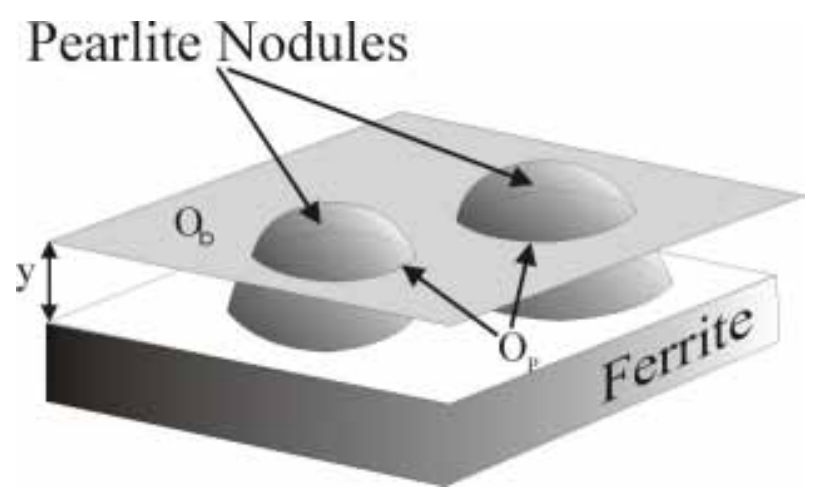

Figure 8

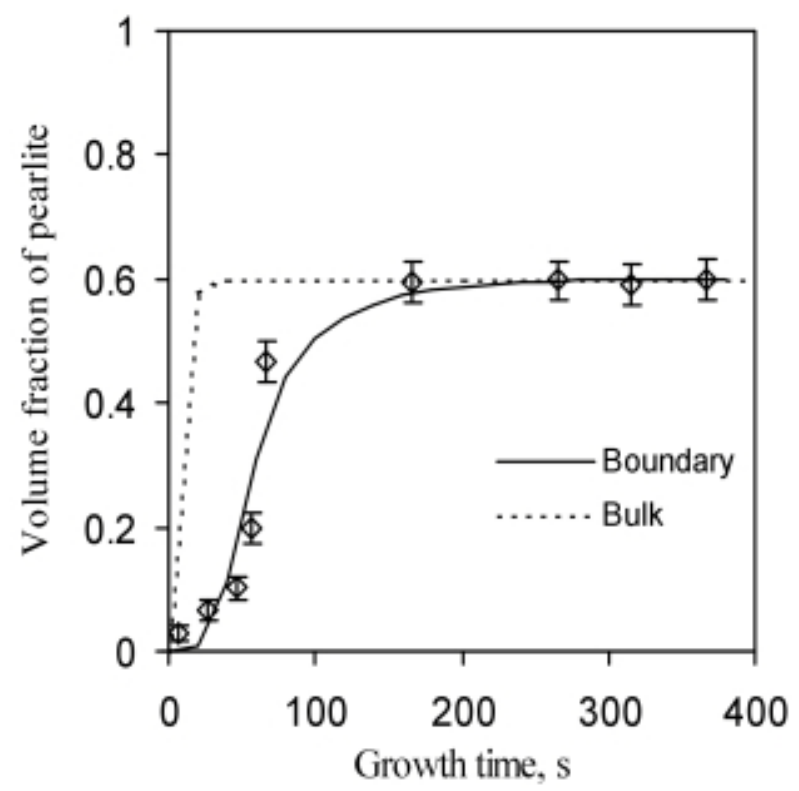

Figure 9(a)

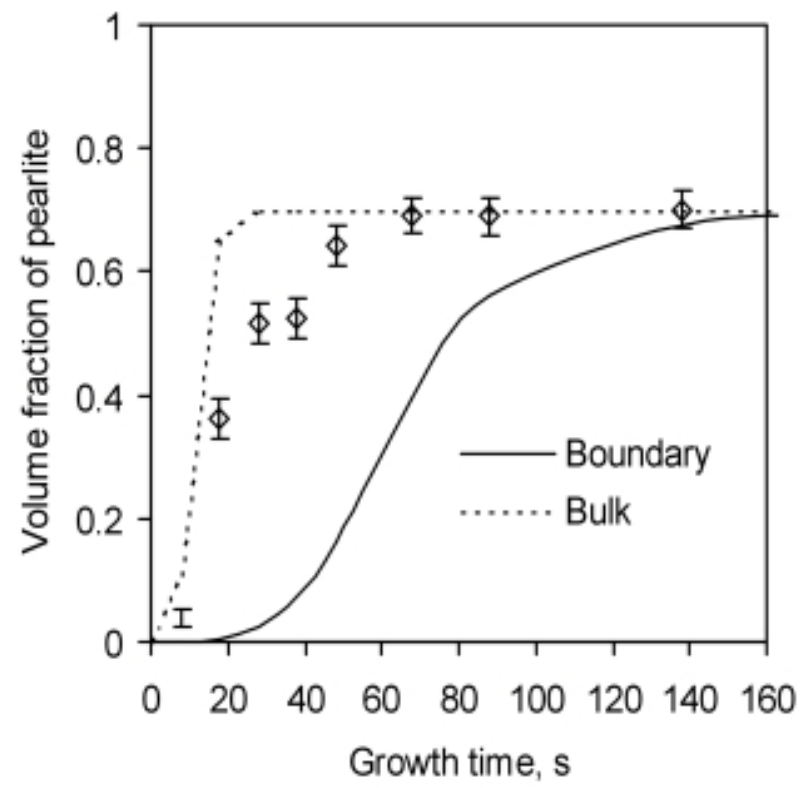

Figure 9(b) 